\title{
Experiencias
}

\section{Adaptación de actividades dirigidas al alumnado con discapacidad visual de primaria mediante la herramienta Scratch en un contexto de confinamiento}

\author{
Scratch tool-mediated adaptation of activities geared \\ to primary school students with visual disability during lockdown
}

\author{
A. G. Molina Riazuelo, E. González Martín
}

\section{Resumen}

La experiencia educativa presentada en este documento nace durante el confinamiento provocado por la enfermedad covid-19 en el curso 2019-2020, y en respuesta a la necesidad de adaptar aquellos ejercicios que los maestros de las aulas proponían para que sus alumnos los realizaran de manera telemática y que, en muchos casos, no resultaban accesibles para el alumnado con discapacidad visual. Para suplir esta carencia, y después de revisar la tipología de las actividades propuestas, se diseñó una batería de «actividades tipo» con el programa Scratch, basada tanto en los contenidos curriculares impartidos por los docentes a distancia, como en las necesidades particulares de los alumnos. Ha sido a partir de este grupo de «actividades tipo», y aprovechando la posibilidad que ofrece Scratch de reutilizar bloques de programación, como se fueron adaptando y programando las diferentes actividades. Tras la puesta en práctica y posterior reflexión sobre dichas actividades con las partes implicadas, se determinó que esta ha sido y es una metodología apropiada en el proceso de generar contenidos educativos accesibles, por lo que se seguirá utilizando para la adaptación de otras actividades en el futuro.

\section{Palabras clave}

Accesibilidad. Covid-19. Enseñanza telemática. Inclusión. Innovación educativa. Tecnología. 


\begin{abstract}
The educational experience described in this article was necessitated by the general lockdown called to combat covid-19 in school year 2019-2020. The experience was undertaken in response to the need to adapt classroom tasks intended to be performed remotely using media that were not accessible to students with visual disability. To overcome that difficulty, after reviewing the type of exercises proposed, a raft of 'standard activities' was designed using Scratch software, based both on the curricular content remotely delivered by educators and on students' specific needs. That series of 'standard activities', together with the reuse of programming units featured by the software, served as grounds for adapting and reprogramming exercises. After Further to activity implementation and subsequent assessment by all the stakeholders, the methodology was determined to be suitable for generating accessible educational content. It will consequently continue to be used to adapt future activities.
\end{abstract}

\title{
Key words
}

Accessibility. Covid-19. ICT-based instruction. Inclusion. Educational innovation. Technology.

\section{Introducción}

En los últimos años, las tecnologías de la información y la comunicación (TIC) han ido tomando fuerza en el campo de la educación, convirtiéndose en una herramienta clave en la escuela. De hecho, el confinamiento provocado por la enfermedad covid-19, iniciado en el mes de marzo de 2020, se convirtió en un catalizador de las TIC, ya que extendió su uso notablemente, proponiendo realizar a los alumnos, la formación académica y diversas actividades online, a través del ordenador y otros dispositivos electrónicos.

La premura con que los maestros tuvieron que acometer este cambio de metodología hizo que, en muchos casos, las actividades propuestas no fueran accesibles para los alumnos con discapacidad visual. ${ }^{1}$

Ante esta situación, cabían dos opciones: o que las familias ayudasen al alumnado ciego en la realización de dichas actividades: leerlas, completarlas, etc., lo cual carecía

1 Este documento utiliza lenguaje no sexista, si bien es cierto que, en algunas ocasiones puntuales, se han utilizado términos genéricos, principalmente plurales, con el único objetivo de otorgar fluidez al texto y facilitar su lectura.

Molina, A.G., y González, E. (2021). Adaptación de actividades dirigidas al alumnado con discapacidad visual de primaria mediante la herramienta Scratch en un contexto de confinamiento. RED Visual: Revista Especializada en Discapacidad Visual, 77, 264-277. https://doi.org/10.53094/HWIV4252. 
de sentido en un contexto en el que se está promoviendo su autonomía, o adaptarlas, de manera que fuesen accesibles y el alumnado con discapacidad visual también las pudiese realizar con la mayor autonomía posible, favoreciendo así, su inclusión.

En este sentido, se pretende encontrar soluciones a este planteamiento a través de la innovación educativa, utilizando, para ello, herramientas lo más accesibles posible que favorezcan la inclusión del alumnado con discapacidad visual. De esta forma, y apoyado en los trabajos realizados por López-Escribano y Sánchez-Montoya (2012), donde se mostró que Scratch era una herramienta que permitía la creación de actividades accesibles para el alumnado con discapacidad visual, surge este proyecto de adaptación de actividades mediante Scratch.

Hay que destacar también que, a la par que se fueron desarrollando estas adaptaciones, se observó que, con este mismo procedimiento, también se podían generar nuevas actividades ajustadas a las necesidades de algunos de los alumnos, creando así actividades de refuerzo personalizadas.

\section{Objetivos}

\subsection{Objetivos generales}

- Adaptar las actividades no accesibles para el alumnado con discapacidad visual, transformándolas en accesibles.

- Diseñar actividades adaptadas a las necesidades de este alumnado.

\subsection{Objetivos específicos}

Relacionados con la programación:

- Elaborar «actividades tipo» de programación sencilla.

- Generar actividades accesibles a partir de las «actividades tipo».

Relacionados con los alumnos:

- Fomentar el manejo de las herramientas tiflotecnológicas.

Molina, A.G., y González, E. (2021). Adaptación de actividades dirigidas al alumnado con discapacidad visual de primaria mediante la herramienta Scratch en un contexto de confinamiento. RED Visual: Revista Especializada en Discapacidad Visual, 77, 264-277. https://doi.org/10.53094/HWIV4252. 
- Desarrollar actividades que fomenten el trabajo autónomo.

- Incluir en las actividades controladores que permitan al alumno comprobar su progreso.

- Trabajar los contenidos educativos a través de la tecnología.

Relacionados con la comunidad educativa:

- Fomentar el trabajo colaborativo entre los miembros de la comunidad educativa.

- Difundir la necesidad de desarrollar actividades teniendo en cuenta los parámetros de accesibilidad.

\section{Público destinatario}

Cabe decir que el grupo de alumnado que participa de estas actividades está compuesto por alumnos de $1 .{ }^{\circ}, 2 .^{\circ}, 3^{\circ}$ y $4 .{ }^{\circ}$ de Educación Primaria que utilizan el braille como código de lectoescritura.

\section{Metodología}

Scratch es un lenguaje de programación especialmente diseñado para iniciarse de manera fácil y sencilla en el mundo de la programación, sin la necesidad de tener conocimientos previos. Sirve para crear historias interactivas, juegos, animaciones... Además, permite el scratching, es decir, la reutilización de los códigos, facilitando la difusión de las creaciones finales con otros usuarios vía Web y, a su vez, la elaboración de nuevos materiales (Pujades, 2016).

De esta forma, este proyecto consistió en elaborar «actividades tipo» que permitieran generar otras actividades similares de forma rápida y con un mínimo de programación. Estas «actividades tipo» permitirían adaptar algunas de las tareas telemáticas propuestas en el aula de forma que fueran accesibles para los alumnos con discapacidad visual, favoreciendo así su inclusión.

Hay que destacar que, con Scratch, dado un juego ya programado, se pueden incorporar o modificar módulos para que se verbalicen las instrucciones. Además, en lugar de manejar los juegos con el ratón, se pueden ajustar para que se utilicen con el teclado. Todo ello, posibilita la participación de los niños con discapacidad visual.

Molina, A.G., y González, E. (2021). Adaptación de actividades dirigidas al alumnado con discapacidad visual de primaria mediante la herramienta Scratch en un contexto de confinamiento. RED Visual: Revista Especializada en Discapacidad Visual, 77, 264-277. https://doi.org/10.53094/HWIV4252. 
En línea con lo anterior, se procuró que las actividades cumplieran lo siguiente:

- Que la interactividad con las actividades fuese a través del teclado del ordenador.

- Que las actividades fueran dirigidas mediante locuciones que aportasen instrucciones e información de las acciones y sus efectos.

- Que durante o al finalizar la actividad, hubiese control de error para que obtuviesen el resultado de esta de manera inmediata.

\section{Materiales y recursos}

Los materiales sobre los que se ha trabajado han sido las propias actividades propuestas por los maestros con su grupo clase: actividades de matemáticas, lengua y conocimiento del medio, generalmente facilitadas a través de archivos PDF o en soporte digital no accesible para alumnos con discapacidad visual.

En este sentido, cabe señalar que esta experiencia se inició en el momento de la pandemia, allá por el mes de marzo de 2020, cuando se impuso el seguimiento telemático de los alumnos. No obstante, este modelo de trabajo se ha continuado durante este curso, en el que, aunque la enseñanza sea presencial, algunas de las tareas que los maestros proponen a sus alumnos requieren modificaciones en relación con la accesibilidad, y con este modelo se facilita.

En relación con los recursos hay que decir que se ha precisado:

- Para el soporte del trabajo: ordenadores y conexión a internet.

- Para la adaptación de estas actividades, como se viene describiendo: Scratch, creando la correspondiente cuenta de usuario.

- Para la coordinación entre los profesionales: Zoom y Teams.

- Para el diseño de actividades: en general, se tomaron como referencia las actividades propuestas por los tutores de aula, pero también se elaboraron algunas personalizadas dirigidas a cubrir las necesidades concretas de los alumnos. 


\section{Descripción de las actividades}

Como se ha comentado, en esta experiencia se revisó el tipo de actividades que se presentaron a los alumnos, observando que estas se podían agrupar en:

- Juegos de teclado.

- Respuesta a preguntas cortas.

- Selección.

- Asociación.

- Elección de respuesta múltiple.

\subsection{Juegos de teclado}

Los juegos de teclado tienen como objetivo que los alumnos se familiaricen con su estructura y manejo. Esto es necesario, ya que interacción con el ordenador por parte de la población con gran disparidad visual se realiza a través de este tipo de dispositivo, ya sea teclado qwerty o línea braille.

En este sentido, se elaboraron algunas actividades encaminadas no tanto al aprendizaje de la mecanografía, como al conocimiento de teclado ya que, en función del dispositivo de entrada (teclado qwerty, línea braille o teclado braille), el alumno debía familiarizase con la posición de las teclas que controlaban el desarrollo de las actividades.

Este grupo de actividades se pueden considerar previas y no todos los alumnos las realizaron, pues ya tenían conocimientos en el manejo de los teclados.

A modo de ejemplo se puede visitar la actividad Juega con el abecedario [recurso web] (instrucciones: pulsar las teclas de las letras; se escuchará el nombre de la letra tecleada).

\subsection{Respuesta a preguntas cortas}

Este grupo de actividades consiste en contestar por escrito a una pregunta. Dentro de este grupo se desarrollaron actividades muy variadas: de ortografía, de ciencias naturales y de inglés.

A modo de ejemplo, se puede visitar las siguientes actividades: 
- Juegos de ortografía [recurso web]. Instrucciones: compuesto por cuatro bloques (palabras con «b», palabras con «V», palabras con «y» y palabras con «X»). Pulsar la bandera para empezar. Después de las instrucciones, se escuchará una palabra. Hay que escribirla en el cuadro de edición y pulsar la tecla «Enter». Si es correcta, se escuchará una nueva palabra. Si se comete un error en la escritura, se escuchará la palabra deletreada y se podrá volver a escribir. Esto se repetirá hasta que se escriba correctamente.

- Animals in English_1 [recurso web]. Instrucciones: pulsar la bandera para empezar. Después de las instrucciones, se escuchará el nombre de un animal, en inglés. Hay que escribirla en el cuadro de edición y pulsar la tecla «Enter». Si es correcto, se escuchará un nuevo nombre. Si se comete un error en la escritura, se escuchará la palabra deletreada y se podrá volver a escribir. Esto se repetirá hasta que se escriba correctamente.

Sirvan de ejemplo estas dos actividades para describir brevemente en qué consiste la reutilización de los códigos de programación en los que se ha basado esta experiencia. En la Figura 1 se muestran unos segmentos de código: a la izquierda, el correspondiente a la actividad Juegos de ortografía y, a la derecha, la reutilización del mismo en la actividad Animals in English_1. Nótese cómo, en ambos casos, la secuencia de bloques de programación es idéntica, cambiando, para generar la segunda actividad, el contenido de la palabra objeto de la actividad, esto es, la palabra «bien» de la actividad de ortografía por la palabra «bear» en la actividad Animals in English_1.

Figura 1: Comparativa de bloques de programación

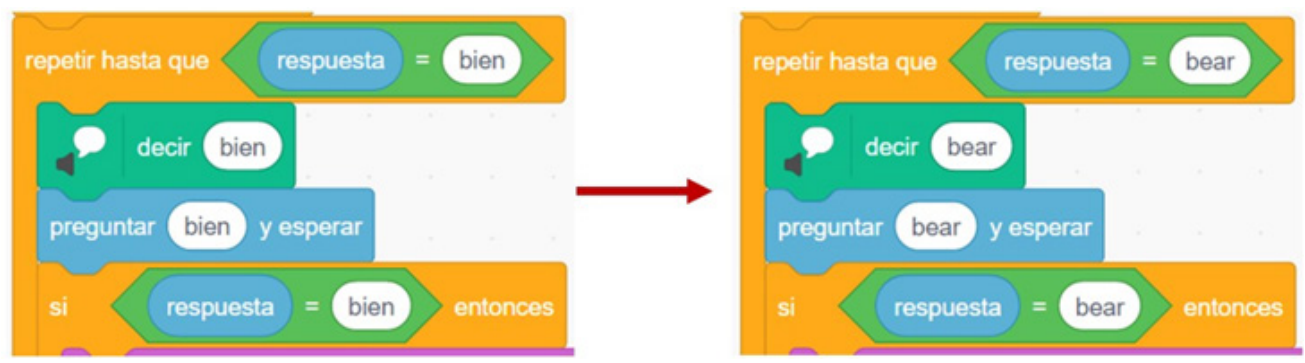

\subsection{Selección}

Estas actividades son de respuesta escrita. Se debe escribir, entre dos propuestas, la correcta. 
A modo de ejemplo se puede visitar la actividad Juegos de ortografía: $b$ / $v$ [recurso web]. (Instrucciones: pulsar la bandera para empezar. Después de las instrucciones se escuchará una palabra que lleve la letra «b» o la letra «v». Hay que escribir la palabra. Si es correcta, se escuchará una nueva palabra. Si se comete un error en la escritura, se escuchará la palabra deletreada y se podrá volver a escribir. Esto se repetirá hasta que se escriba correctamente).

Esta actividad tipo se desarrolló a demanda, para cubrir las necesidades que una alumna tenía, concretamente, en ortografía. Ha servido de modelo para desarrollar otras similares.

Se pueden consultar los bloques de programación a través del botón «Ver dentro» que se encuentra en la propia actividad.

\subsection{Actividades de asociación}

En estas actividades, se proponen dos columnas de elementos que deben asociarse. Habitualmente, se representan gráficamente uniéndolos con una línea. Para facilitar la tarea mediante teclado, la actividad se modificó un poco. Las palabras de la columna de la derecha se presentaban de una en una mientras que la columna de la izquierda se mostraba completa.

Este grupo de actividades se realiza mediante las flechas del teclado.

A modo de ejemplo, se puede visitar la actividad Vertebrados: características [recurso web], que es una adaptación de la actividad interactiva Características de los vertebrados del libro digital Ciencias de la Naturaleza 4 Primaria propuesta por la editorial Anaya (Gómez y Valbuena, 2019, p. 47). (Instrucciones: escuchar el nombre de un grupo de animales vertebrados; recorrer con la flecha hacia arriba o hacia abajo las características y seleccionar con la flecha derecha la que corresponda).

\subsection{Elección de respuesta múltiple}

Se incluyen en este grupo aquellas actividades en las que se formula una pregunta y el estudiante debe elegir la respuesta correcta entre varias opciones.

Este tipo de actividades requiere un poco más de programación ya que lleva varios grupos de bloques. Una vez diseñadas, al igual que en los ejemplos

Molina, A.G., y González, E. (2021). Adaptación de actividades dirigidas al alumnado con discapacidad visual de primaria mediante la herramienta Scratch en un contexto de confinamiento. RED Visual: Revista Especializada en Discapacidad Visual, 77, 264-277. https://doi.org/10.53094/HWIV4252. 
anteriores, se pueden generar, a partir de ellas, nuevas actividades, aunque requieren un poco más de atención, ya que hay que modificar el contenido de varios bloques.

A modo de ejemplo se pueden consultar las siguientes actividades:

- Luis y las fracciones [recurso web] (instrucciones: juego de preguntas sobre fracciones con varias opciones de respuesta; escuchar las instrucciones y seguir el juego). Juego de preguntas basado en la actividad interactiva Contenidos básicos: sumar fracciones del libro digital Matemáticas 4 Primaria: más savia (Garín et al., 2019).

- Palabras homófonas remix [recurso web] (instrucciones: escuchar las instrucciones y seguir el juego). Juego de preguntas basado en la actividad interactiva Palabras homófonas del libro digital de Lengua 4 Primaria (Burruezo et al., 2019, p. 79), versionado para adaptarlo a las necesidades del alumnado con discapacidad visual. Se pueden consultar los bloques de programación a través del botón «Ver dentro» que se encuentra en la propia actividad.

\section{Trabajo colaborativo}

En el desarrollo de esta experiencia han participado tanto profesionales como familias. Unos se han encargado de la selección de actividades, otros de la programación y adaptación, y otros de su validación. Todo ello ha supuesto una interesante experiencia de colaboración que se puede describir como sigue:

- Los profesores de aula trasladan las actividades propuestas para el grupo clase a la maestra de apoyo del Equipo Específico de Discapacidad Visual.

- La maestra de apoyo y la maestra de Accedo-Madrid (Accesibilidad a Contenidos Educativos de la ONCE) se coordinan para revisar, clasificar y priorizar las actividades.

- Se programan las «actividades tipo».

- Se revisan añadiendo mejoras, cuando corresponda.

Molina, A.G., y González, E. (2021). Adaptación de actividades dirigidas al alumnado con discapacidad visual de primaria mediante la herramienta Scratch en un contexto de confinamiento. RED Visual: Revista Especializada en Discapacidad Visual, 77, 264-277. https://doi.org/10.53094/HWIV4252. 
- A partir de la «actividad tipo», se crean nuevas actividades (de acuerdo con las propuestas de los maestros del aula), adaptando los bloques de programación.

- Se difunden las actividades tanto a los profesores de aula como a las familias.

- Las observaciones y comentarios que aportan tanto los niños como los profesionales y familias facilitan la retroalimentación del proceso, lo que contribuye a aumentar la calidad de las actividades y la posterior creación de otras nuevas.

\section{Resultados}

Desde el punto de vista de las autoras que han participado en esta experiencia, los resultados obtenidos han sido, por lo general, muy positivos, destacando lo siguiente:

- Scratch permite adaptar actividades para hacerlas accesibles a alumnos con discapacidad visual.

- Su estructura en bloques permite que, a partir de un modelo, intercambiando dichos bloques, se puedan generar fácilmente nuevas actividades.

- Scratch incluye bloques que trascriben texto a voz, lo que facilita el diseño y desarrollo de actividades accesibles para la población con discapacidad visual.

- El hecho de poder diseñar actividades para realizar mediante teclado permite, a su vez, interaccionar con ellas a través de la línea braille.

- El manejo con teclado permite la entrada desde dispositivos móviles, teléfono o tableta, a través de teclado qwerty o la línea braille, conectados vía Bluetooth.

- Este tipo de ejercicios promueven la competencia digital del alumnado, lo que, además, favorecerá su inclusión social y académica, cumpliendo así con las Directrices Educativas de la Dirección de Educación, Empleo y Braille de la ONCE.

Sin embargo, también es importante reseñar algunos aspectos a considerar, en relación con el manejo de estas actividades generadas con Scratch, para el alumnado con discapacidad visual: 
- Scratch requiere de un auxiliar que guíe al alumno hasta el inicio de la actividad. A partir de ahí, ya las podrá desarrollar con plena autonomía.

- La programación de estas actividades no es accesible para personas que precisen el uso de revisores de pantalla para la navegación.

Por otro lado, y tras un periodo oportuno de uso de las actividades realizadas por parte de los destinatarios de esta experiencia, se ha reflexionado junto a ellos para evaluar los resultados. De esta manera:

- Los niños han indicado que, por lo general, las actividades son fáciles de utilizar, son muy entretenidas y les han gustado mucho.

- Las familias han indicado que estas actividades permiten a los niños realizar ciertas tareas que, de otro modo, no podrían haber hecho si no hubiese sido con su ayuda directa. Además, les permite trabajar y reforzar indirectamente el uso del teclado de manera lúdica en un contexto en el que se han tenido que suspender las clases de Iniciación a la informática y editor de textos con comandos de teclado. Sin embargo, en algunas ocasiones, al ejecutar un comando sin haber escuchado la locución al completo se han solapado varios audios, dificultando su realización.

- Los docentes de los centros educativos son conocedores de la dificultad que suponen, para los alumnos y sus familias, las actividades que no son accesibles. Por ello, han apreciado el esfuerzo realizado en la adaptación de las actividades propuestas. De hecho, en algunos casos, dichas actividades han sido realizadas a modo de repaso por todos los alumnos de la clase. Además, al tener que utilizar el teclado, ayuda a los alumnos a trabajar la competencia digital a través de las TIC, área de intervención clave en el alumnado con discapacidad visual.

\section{Discusión}

Innegablemente, la tecnología facilita crear contenidos accesibles para el alumnado en general $y$, concretamente, para el alumnado con discapacidad visual, supliendo las carencias de algunos materiales proporcionados en los cen- 
tros educativos. De esta forma, Scratch ha permitido su adaptación, influyendo positivamente en el proceso de enseñanza-aprendizaje con este alumnado, en un contexto de escuela telemática obligado por el confinamiento causado por la pandemia.

Se ha constatado que los alumnos son bastante autónomos con esta herramienta. De hecho, Fernández et al. (2017) indican que las TIC favorecen la autonomía del alumnado con discapacidad, aunque necesitan un periodo de tiempo de exploración y adaptación a las mismas. Otros factores clave que destacan estos autores acerca de las TIC en general, y que se pueden aplicar a Scratch en particular, son su capacidad de adaptación a las necesidades particulares de cada alumno, así como la capacidad de ofrecer la respuesta del ejercicio de manera inmediata, aspectos clave de este trabajo.

Sin embargo, y como se ha mencionado anteriormente, en este caso en concreto el alumnado necesita de la colaboración de un adulto para dar comienzo a la actividad, ya que este paso carece de accesibilidad. No obstante, al tratarse de alumnos de corta edad que requieren igualmente de la ayuda de un adulto para abrir la actividad, este hecho no ha sido determinante, considerándose, de todas formas, a Scratch como una herramienta apropiada por las posibilidades que ofrece.

Por otro lado, la facilidad de creación de nuevas actividades, una vez que se tuvieron diseñadas las «actividades tipo», permitió la creación de nuevos contenidos ajustados a las necesidades específicas de nuestro alumnado.

Merece la pena señalar el interés despertado en los alumnos por las actividades generadas mediante el lenguaje de programación Scratch. Este aspecto es muy importante ya que la motivación facilita que el alumno se involucre con mayor interés, repercutiendo positivamente en su posterior aprendizaje, lo cual coincide con los resultados obtenidos por Durango-Warnes y Ravelo-Méndez (2020).

Por último, señalar que, aunque esta experiencia ha surgido en respuesta a la necesidad que los maestros de centro tenían por disponer de materiales accesibles para trabajar telemáticamente con sus alumnos con discapacidad visual, dados los resultados y la valoración por parte de los diferentes miembros de la comunidad educativa, se estima que esta experiencia se extenderá más allá del momento concreto de pandemia en el que se inició. 


\section{Propuestas de mejora}

En algunas ocasiones, al ejecutar comandos sin que la locución hubiera finalizado, se iniciaba la siguiente, solapándose varios audios y dificultando la realización de la actividad. En este aspecto ya se está trabajando mediante la modificación de la programación para su mejora.

Aunque esta actividad ha venido a paliar la necesidad de un momento educativo muy específico, aún es necesario reforzar con algunos docentes de los centros educativos la idea de que la inclusión reside en la utilización de los mismos recursos adaptados a las necesidades del grupo-clase, no en la adjudicación de una tarea adaptada para un alumno en concreto. Este aspecto se tiene que seguir trabajando en el día a día.

\section{Conclusiones}

Tras la interpretación y reflexión de los resultados obtenidos, se ha llegado a las siguientes conclusiones:

- Scratch permite la adaptación de actividades no accesibles y la creación de nuevos materiales inclusivos.

- Destacar la importancia del trabajo colaborativo entre los diferentes miembros de la comunidad educativa.

- Es fundamental la formación continua de los docentes, específicamente en el área de tecnología, para que la escuela se adapte a las necesidades surgidas en un contexto de cambio como el que nos ha sobrevenido, sin que se vea afectada la inclusión educativa.

\section{Referencias bibliográficas}

Burruezo, T., Martínez, S., Moreno, A. P., y Tolsada, S. (2019). Lengua 4 Primaria: pieza a pieza. Anaya.

Durango-Warnes, C., y Ravelo-Méndez, R. E. (2020). Beneficios del programa Scratch para potenciar el aprendizaje significativo de las Matemáticas en tercero de primaria. Trilogía Ciencia Tecnología Sociedad, 12(23), 163-186. https://doi.org/10.22430/21457778.1524.

Molina, A.G., y González, E. (2021). Adaptación de actividades dirigidas al alumnado con discapacidad visual de primaria mediante la herramienta Scratch en un contexto de confinamiento. RED Visual: Revista Especializada en Discapacidad Visual, 77, 264-277. https://doi.org/10.53094/HWIV4252. 
Fernández, J.M., Román, P., y El Homrani, M. (2017). TIC y discapacidad: conocimiento del profesorado de educación primaria en Andalucía. Aula Abierta, 46, 65-72. https:// doi.org/10.17811/rifie.46.2017.65-72.

Garín, M., Vidal, J.M., Sánchez, P., Morales, F., Bernabéu, J., Pérez, M. N., Hidalgo, J.M., Moratalla, V., y Cabello, M. F. (2019). SD Profesor: Matemáticas 4 Primaria: más savia. SM.

Gómez, R., y Valbuena, R. (2019). Ciencias de la Naturaleza 4 Primaria: pieza a pieza. Anaya.

López-Escribano, C., y Sánchez-Montoya, R. (2012). Scratch y Necesidades Educativas Especiales: programación para todos [PDF]. Revista de Educación a Distancia (RED), 34.

Pujades, N. (2016). ¿Qué es Scratch? Scratch School. https://www.scratch.school/ aprender/que-es-scratch/\# : : text=Scratch\%20es\%20un\%20lenguaje\%20de,de\%20 forma\%20intuitiva\%20mediante\%20bloques.

Ana Gloria Molina Riazuelo. Maestra de Educación Infantil. Miembro de Grupo Accedo-Madrid. Centro de Recursos Educativos de la ONCE en Madrid. Avenida del Doctor García Tapia, 210; 28030 Madrid (España). Correo electrónico: agmr@once.es.

Esther González Martín. Maestra del Equipo Específico de Discapacidad Visual. Centro de Recursos Educativos de la onCE en Madrid. Avenida del Doctor García Tapia, 210; 28030, Madrid (España). Correo electrónico: esgom@once.es. 